\title{
Monográfico: Adaptación y accesibilidad de las tecnologías para el aprendizaje
}

\author{
Jesús G. Boticario \\ Olga C.Santos \\ Alejando Rodríguez Ascaso
}

(Coordinadores) 



\title{
PRESENTACIÓN
}

\section{ADAPTACIÓN Y ACCESIBILIDAD DE LAS TECNOLOGÍAS PARA EL APRENDIZAJE}

\author{
Jesús G. Boticario, Olga C. Santos y Alejandro Rodríguez Ascaso \\ (coordinadores) \\ Grupo de Investigación aDeNu \\ Dpto. Inteligencia Artificial, UNED, España.
}

La adaptación aplicada a la educación mediada por tecnología ha dejado de ser una cuestión de interés científico centrada en el desarrollo de pequeños prototipos para convertirse en un aspecto primordial, sin el cual es difícil abordar los retos actuales planteados en todos los niveles educativos, pero especialmente en el campo de la educación superior y el aprendizaje permanente (AP, que responde al conocido término en inglés "Lifelong Learning"). El reto planteado es, tal y como se ha expuesto en numerosos informes e iniciativas, el aprendizaje centrado en el estudiante y sus necesidades, así como el tratamiento de su evolución a lo largo del tiempo.

En este contexto, se supone que la formación universitaria ha de favorecer un aprendizaje flexible, regido por criterios fiables y justificados, planteando una visión del conocimiento como proceso constructivo en el que es esencial facilitar el aprendizaje de nuevas competencias docentes. Bajo esta perspectiva, se asume que el aprendizaje de los estudiantes debe estar sujeto a un proceso constructivo, activo, autorregulado, situado y social, en el que la participación del sujeto en el proceso es imprescindible. En definitiva, se reafirma que el proceso de aprendizaje es un proceso personal y centrado en el individuo que afecta a sus competencias y habilidades y se devalúan las perspectivas centradas en el mero aprendizaje de un determinado cuerpo de conocimientos.

El paradigma del AP considera que el aprendizaje debe realizarse a lo largo de la vida de las personas, de forma que puedan integrarse educación, trabajo y vida personal en un proceso continuo que considera el aprendizaje reglado, el informal -hoy en día de especial interés por el uso creciente de las redes sociales - y el ámbito laboral. En el mismo se asume que la educación debe ser inclusiva, lo que implica que la tecnología debería asegurar el tratamiento de la diversidad de necesidades y el cumplimiento de los requisitos de accesibilidad desde cualquier lugar y en cualquier contexto. 
El uso de los estándares y especificaciones relacionados con la educación y la interacción con las personas deberían garantizar la sostenibilidad de los desarrollos y la interoperabilidad con todos los elementos implicados: usuarios, contenidos, actividades, dispositivos de acceso, etc. Estos estándares deberían permitir el modelado de escenarios personalizados que consideren las características individuales del estudiante y su entorno.

De otra parte, se observa un creciente desarrollo de nuevos marcos y entornos abiertos de aprendizaje centrados en el uso de arquitecturas de servicios web y herramientas basadas en la web semántica, con los que se espera facilitar la interoperabilidad de las aplicaciones, su reutilización y sostenibilidad.

Todo lo expuesto plantea numerosos desafíos de interés que abarcan temas diferentes relacionados con el modelado del usuario y de la instrucción, del contexto, la colaboración, el uso de los nuevos estándares disponibles centrados en las necesidades de interacción de las personas, la aplicación de sistemas recomendadores y de técnicas de minería de datos en la educación, entornos de aprendizaje personalizado, la accesibilidad y usabilidad de todos los recursos utilizados, escrutabilidad y acceso a los modelos abiertos de los estudiantes, el desarrollo de arquitecturas abiertas de servicios educativos, etc.

El objetivo de este número especial de RIED es mostrar los avances tecnológicos, en los campos mencionados y en otros relacionados, que se están realizando para garantizar un aprendizaje adaptativo e inclusivo centrado en el estudiante y en sus competencias, que atienda su diversidad funcional, considerando el interés de poder abordar la evolución de dicho aprendizaje a lo largo del tiempo.

Los artículos incluidos en este número especial pueden agruparse en torno a diversos temas de interés: I) elementos estructurales que soportan la adaptación y la accesibilidad de los contenidos y las interacciones del estudiante con el sistema, II) cuestiones e infraestructura de apoyo al diseño para todos que permiten describir las necesidades de los usuarios y sus preferencias de interacción y de carácter psicoeducativo, III) aplicaciones y soluciones en tiempo de ejecución que facilitan la interacción personalizada y accesible con los contenidos y los servicios ofrecidos y, finalmente, IV) herramientas para verificar la usabilidad de las soluciones desarrolladas para atender la diversidad funcional de los usuarios.

Dentro de los elementos estructurales, en el trabajo de Fernando Alonso y otros se presenta una recopilación actualizada de los principales estándares relacionados 
con la accesibilidad, usabilidad y adaptabilidad de los sistemas de e-Learning. El análisis es exhaustivo y cubre modelos de usuario, escenarios de aprendizaje, preferencias de interacción, capacidades de los dispositivos y metadatos para especificar cómo entregar cualquier recurso de acuerdo a las necesidades de los usuarios, así como otros estándares relevantes en el campo de la accesibilidad y la usabilidad del software. Del estudio se concluye que hay una falta de estándares que estén orientados al mismo tiempo hacia los usuarios y los desarrolladores y que tengan en cuenta todas las áreas de modelado.

Los elementos estructurales requeridos para desarrollar sistemas adaptativos y accesibles son revisados en el artículo de David Mérida y otros. Una vez clarificada la naturaleza de los sistemas adaptativos se repasan los elementos básicos del modelado. En concreto, se incide en las propuestas planteadas para resolver el problema del modelado del contexto del usuario y de los contenidos a adaptar, así como los de la interacción entre el usuario, los contenidos y el contexto, analizando los estándares y herramientas (p.ej., ontologías), que sirven para hacer operativos, interoperables y reutilizables las soluciones aportadas. Se muestran ejemplos concretos de cómo se especifican estos desarrollos mediante estándares y se introduce una arquitectura multiagente capaz de llevar a efecto las adaptaciones señaladas.

Con un planteamiento basado en el diseño para todos, que garantiza el considerar las cuestiones de diversidad funcional desde el comienzo del proceso de desarrollo, Covadonga Rodrigo y otros nos muestran el uso de formatos contenedores para desarrollar recursos multimedia, lo cual redunda en una mejora de los actuales procesos de producción, distribución, y mantenimiento de los contenidos y escenarios educativos así como amortiza el esfuerzo realizado asegurando la mayor reutilización e interoperabilidad de los mismos. En este trabajo se resaltan aquellos aspectos que deben contemplarse para garantizar la accesibilidad de los formatos y lograr adaptar el uso de los contenidos a las necesidades del estudiante.

Igualmente con un enfoque centrado en atender las necesidades del individuo desde el comienzo del diseño de la experiencia educativa y en poder reutilizar las soluciones mediante el uso de estándares, en el artículo de Elena del Campo y otros se muestra el desarrollo de diseños instruccionales (construidos sobre la especificación IMS Learning Design) que tienen en cuenta distintos tipos de estudiantes que presentan diferentes necesidades de apoyo psicoeducativo (p.ej., déficit de atención, dislexia y déficits auditivos). Se presenta la elaboración de un curso adaptativo (esto es, adaptable en tiempo de ejecución en función de las interacciones del usuario) centrado en la familiarización del estudiante con el entorno virtual de aprendizaje, 
considerando el diseño, implementación e integración de las unidades de aprendizaje con los servicios de apoyo necesarios en la plataforma de aprendizaje, lo que permite que las adaptaciones preparadas puedan aplicarse al estudiante en un entorno educativo real.

Para ilustrar aplicaciones concretas que tratan con los problemas de la adaptación, Álvaro Ortigosa y otros describen sistemas que dan soporte a la creación y evaluación de entornos de aprendizaje móviles adaptativos. En ellos se recomiendan las actividades más adecuadas para cada estudiante en cada momento y se adapta la interfaz, seleccionando los contenidos y herramientas más apropiados en cada caso. Dada la complejidad de evaluar la adecuación para cada usuario de las recomendaciones y adaptaciones se presenta un sistema que utiliza técnicas de minería de datos para extraer de las interacciones de los estudiantes información sobre los elementos que causaron mayores dificultades. Estos problemas se muestran al profesor, de modo que éste pueda intervenir añadiendo/eliminando/ modificando actividades, contenidos o criterios de recomendación o adaptación.

Ahondando en las capacidades de los estándares y especificaciones para el diseño de escenarios de educación adaptativos, en concreto, de nuevo mediante la especificación IMS Learning Design, en el artículo de Derick Leony y Luis de la Fuente se presenta una recopilación de experiencias basadas en la creación e instanciación de estrategias adaptativas basadas en el uso de dicho estándar, y cuyo objetivo es la determinación empírica de la expresividad de la especificación en el ámbito de la adaptación de materiales educativos. En este trabajo se discuten las relaciones entre accesibilidad, adaptación y flexibilidad, abordando en concreto los temas de accesibilidad de contenidos y del sistema en sí, así como los problemas derivados de la especificación y de la herramienta que permita desarrollar escenarios educativos según dicha especificación.

Una alternativa de interés creciente para el desarrollo de sistemas de educación adaptativos es utilizar los llamados sistemas recomendadores. En el trabajo de Priscila Valdiviezo y otros se discute el uso, mediante técnicas de minería de datos, de los datos de interacción de los estudiantes para identificar elementos de interés que permitan ofrecer las recomendaciones adecuadas a cada estudiante y situación. Aquí se describe la identificación de patrones comunes de interacción en los foros disponibles en un curso para mejorar la elaboración de dichas recomendaciones. Mediante técnicas de agrupación aplicadas sobre indicadores de colaboración relevantes como nivel de iniciativa, de actividad y de reacciones causadas por un usuario, se permite caracterizar a los estudiantes como participativos, no 
colaborativos y con iniciativa. Partiendo de la detección automática de estas situaciones se plantea la redefinición de recomendaciones para cada tipo de usuario alimentando un modelo de recomendaciones que soporta la descripción de dichas recomendaciones mediante técnicas de diseño centrado en el usuario.

Finalmente Jaime Sánchez nos muestra una metodología para desarrollar y evaluar la usabilidad de entornos virtuales basados en audio para el aprendizaje y la cognición de usuarios ciegos. El sonido espacial -también llamado sonido 3Dpuede ayudar a mejorar y ejercitar la memoria, la percepción háptica, las estructuras cognitivas temporales y espaciales, la orientación y movilidad, el aprendizaje de las matemáticas y ciencias, y la resolución de problemas. En esta investigación se identifican instrumentos claves para una correcta evaluación de usabilidad y cognitiva de un entorno basado en sonido para usuarios con discapacidad visual. Aplicando la metodología se repasan entornos virtuales basados en audio. Las evaluaciones de usabilidad confirman las ventajas operativas y cognitivas de los entornos virtuales con sonido espacial para los usuarios no videntes.

Como editores de este número especial queremos agradecer a los responsables de la revista RIED su apoyo para su elaboración, a los autores de las contribuciones presentadas, su involucración en la escritura de artículos centrados en los temas tratados en la revista y, en particular, al apoyo recibido de los proyectos de investigación nacionales y europeos del grupo de investigación aDeNu de la UNED que sufragan el desarrollo de sistemas accesibles y adaptativos de educación, tales como EU4ALL (ISTFP6-034778), CISVI (TSI-020301-2008-21), ACCEDO2.0 (TSI040200-2009-137) y muy especialmente A2UN@ (TIN2008-06862-Co4-0o/TSI).

Jesús G. Boticario, Olga C. Santos y Alejandro Rodríguez Ascaso

(Coordinadores)

Grupo de Investigación aDeNu, Dpto. Inteligencia Artificial, UNED

Madrid, España. 